\title{
Reviewing off-axis telescope concepts A quest for highest possible dynamic range for photometry and angular resolution
}

\author{
Gil Moretto $^{*(1)}$, Jeff R. Kuhn ${ }^{(2)}$ and Phil R. Goode ${ }^{(3)}$ \\ (1) Lyon Institute of Origins, Institute of Nuclear Physics of Lyon, IN2P3-CNRS, \\ Université de Lyon, F-69622, Lyon, France \\ (2) Institute for Astronomy, University of Hawaii, Honolulu, Hawaii, USA \\ (3) Big Bear Solar Observatory, NJIT, Big Bear City, California, USA
}

\begin{abstract}
We review off-axis telescope concepts that use unobstructed pupils. Built and prospective telescopes for ground and space astronomy will be presented and discussed. Such concepts offer great advantages in terms of emissivity, throughput, diffraction- limited energy concentration and higher dynamic range. The coronagraphic performance of off-axis telescopes will enable instruments, which are starving for higher dynamic range, for example, those devoted to faint companion detection and solar studies. Smaller telescopes like SOLAR-C (IfA/Haleakala Observatory), and the New Solar Telescope (NST/NJIT/ Big Bear Observatory) are operational and are test beds for the Advanced Technology Solar Telescope (ATST Project) for which site construction is beginning on Haleakala.
\end{abstract}

Keywords: off-axis telescope, low scattered light, low emissivity, higher dynamic range

\section{INTRODUCTION}

We address here off-axis telescope concepts with its advantages in terms of emissivity throughput, diffraction-limited energy concentration and higher dynamic range. The combination of these advantages can make a medium size telescope scientifically competitive with large instruments.

With a review of off-axis telescopes already built and proposed concepts, we expose several myths about the feasibility of off-axis telescopes and address the question of why we need off-axis telescopes. The assessment of new technologies count on the off-axis telescopes already built, for example SOLARC and NST. Such projects act as pathfinders for future off-axis telescope projects improving the maturity of evolving technologies; enabling more thoroughly understand how to overcome the technical and engineering challenges specific to this kind of design.

The polishing of the first $8.4 \mathrm{~m}$ parabolic off-axis mirror by the Steward Observatory Mirror Lab (SOML) is also a fundamental milestone for which the NST was a pathfinder. The SOML is not alone, several other fabricators (Reosc/France) and university labs (UNAM/Mexico) are routinely polishing off-axis optics.

* G. Moretto: E-mail: Gil.Moretto@ipnl.in2p3.fr, Telephone: 33 472.431.108

Ground-based and Airborne Telescopes IV, edited by Larry Stepp, Roberto Gilmozzi, Helen J. Hall,

Proc. of SPIE Vol. 8444, 8444OY • (C) 2012 SPIE • CCC code: 0277-786/12/\$18• doi: 10.1117/12.926780 


\section{ADVANTAGES OF OFF-AXIS TELESCOPES}

It has been shown that telescope systems making use of a conventional obstructed on-axis concentric designs, can be limited by the scattered light and emissivity caused by the auxiliary mirrors.

Figure 1 shows a representative image of a bright star obtained with a conventional $4 \mathrm{~m}$ aperture astronomical telescope. Most of the diffuse structure in the image is the result of spurious scattered and diffracted light from the material obstruction of the pupil associated with the secondary mirror. The faint radial flares near the center of the image are caused by scattering from the primary and secondary mirrors, while much of the scattered light haze in the image core is due to atmospheric scatter.

A more detailed analysis for the relative contribution of various scattered light sources for a conventional telescope has been described by Kuhn and Hawley (1999) and more specifically for a $6.5 \mathrm{~m}$ telescope at a wavelength of 1 and 4 microns by Moretto \& Kuhn (2000).

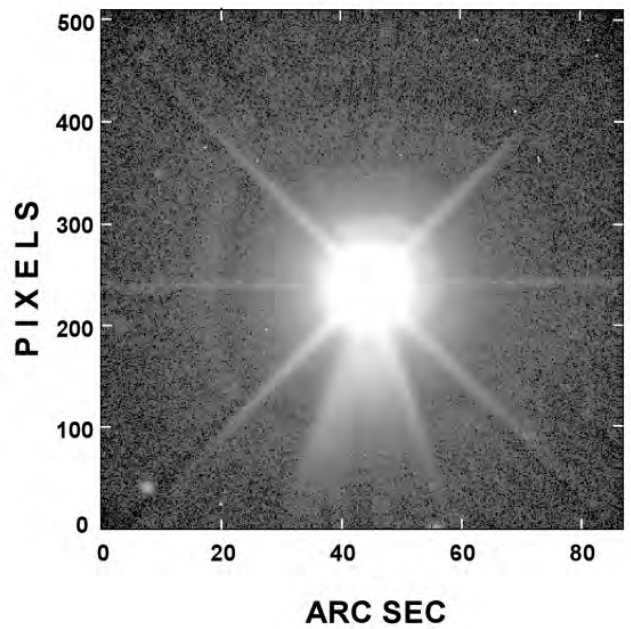

Figure 1 - A representative bright star image obtained with a conventional $4 \mathrm{~m}$ aperture obscured astronomical telescope (CTIO Blanco $4 \mathrm{~m}$ ). Part of sky is hidden from our point of view by scattered light. The display range shows 3 orders of magnitude and is logarithmic. (Moretto and Kuhn, 2000

Figure 2(A) shows in more detail each PSF contribution for a $6.5 \mathrm{~m}$ at a wavelength of 1 micron. Figures 2(B) and 2(C) show how the relative importance of edge (aperture) diffraction to the scattered surface brightness increases with wavelength and dominates the telescope PSF at wavelengths of a few microns over field angles ranging from a few arcseconds to several arcminutes.

Such light scattering characteristics of the telescope will limit the telescope performance for measurements requiring very high photometric dynamic range, for example astronomical observations of faint objects near bright sources. In order to extend telescope scientific capabilities and performance light scattering should be controlled and minimized. How? The ways to do so, is through (i) to use unobstructed pupil (off-axis) telescope; eliminating the spider diffraction contribution to the telescope PSF (Figure 2); and (ii) by improving the telescope mirrors optical quality; minimizing mirror scatter contribution (Figure 2(B) and 2(C)).

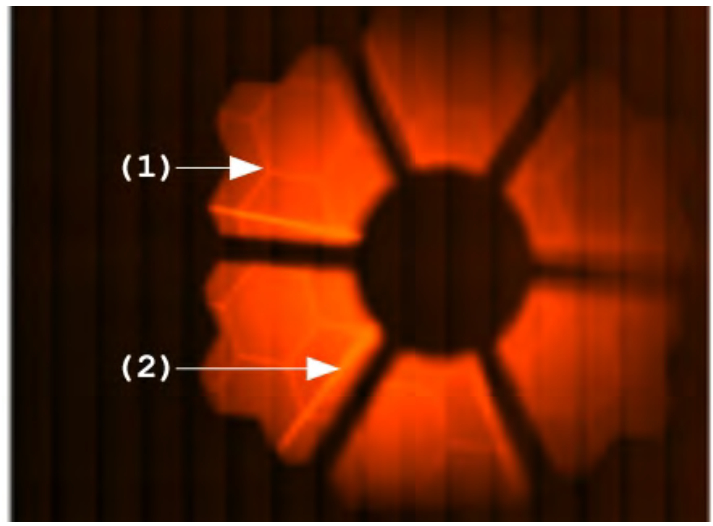

Figure 3 - Image of the Gran Telescopio Canarias (GTC) telescope pupil showing some of the warm components of telescope emission; (1) is emission from the interspaces between M1 segments and (2) from the spider suppor structure. The 'Rose Petal" Lyot mask within CanariCam was used. 


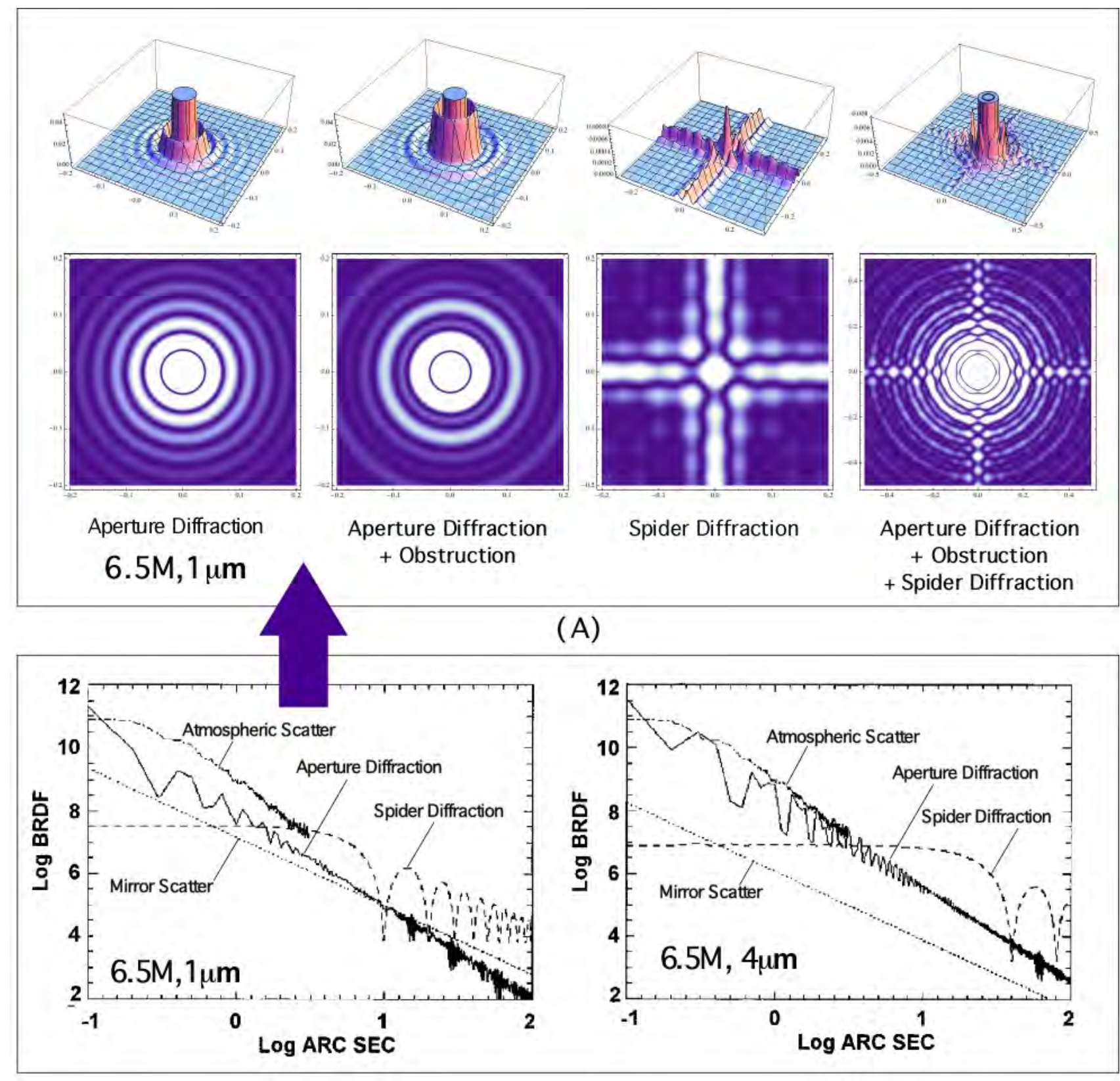

(B)

(C)

Figure 2 - (A) The diffraction patterns $(\lambda=1 \mu \mathrm{m})$ along the orthogonal $\theta x$ - or $\theta y$-directions; (up) the 3D and (down) the density profiles for the $6.5 \mathrm{~m}$ unobscured aperture (edge) diffraction, aperture diffraction with obscuration $(\varepsilon=0.250)$, spider diffraction along the orthogonal legs (4 legs 50mm x 3250mm), and finally all PSF diffraction contributions. (B) and (C) are the scattered light PSF contributions for a conventional $6.5 \mathrm{~m}$ telescope at 1 micron and 4micron. The solid line shows the unobscured aperture (edge) diffraction. The dotted line shows the BRDF from mirror roughness scattering assuming a mirror as smooth as the Hubble Space Telescope primary. The dashed line shows the BRDF from a $2 \mathrm{~cm}$ wide secondary mirror support spider and the dash-dotted line shows the atmospheric BRDF for an atmosphere characterized by a $15 \mathrm{~cm}$ Fried parameter. 
Comparing Figures 2(B) and 2(C) it is even more important the advantages of an unobstructed pupil for thermal infrared optimized telescopes. Also an off-axis telescope reduces the sources of self thermal emissivity. Figure 3 shows the image of the Gran Telescopio Canarias (GTC) telescope pupil showing some of the warm components of the telescope emission. In light gray color we can see the emission from the interspace between M1 segments (1) and from the M2 support structure spider (2).

Another advantage inherent to off-axis telescopes is the absence of azimuthal PSF structure; no missing or interpolated wavefront errors; a natural advantage for interferometry and adaptive optics performance.

\section{THE PATHFINDERS SOLAR-C AND NST}

We present here off-axis telescopes projects whose had been built. Such projects have served as pathfinder prototypes to larger telescopes projects in prospective and underway.

SOLARC - Scatter-free Observatory for Lim Active Regions and Coronae is a 0,5m off-axis coronagraphic reflecting telescope located on Haleakala (Hawaii) adjacent to Mees Solar Observatory. Construction was started in October 1998 and first light (Figure 4) was achieved in August 2001. The scientific goals are (i) provide more accurate coronal measurements which have not been realized, even from space, in order to explain and better understand many solar phenomena; (ii) support several long-term coronal observing platforms which extend intermittent coronal space observations. SOLARC has acted as a prototype off-axis telescope for others projects such as NST and ATST (presented later) in order to provide a model environment for the future ATST project and to enable more thoroughly understand how to overcame the technical and engineering challenges specific to this kind of telescope design: alignment, heat dissipation and guiding off of asymmetrical phenomena.

The pioneer SOLARC has demonstrated some of off-axis telescope "myths": aberrations are not worse than conventional telescopes; off-axis telescopes can be aligned and off-axis mirrors are manufacturable.

NST - New Solar Telescope is the largest aperture and highest resolution solar telescope. The $1.6 \mathrm{~m}$ off-axis primary mirror housed at the Big Bear Solar Observatory (BBSO), in California. NST is a collaboration of BBSO/NJIT with the University of Hawaii and the Korea Astronomy \& Space Science Institute. The NST used and employed the SOLARC "savoir-faire” for its realization. See Goode et al (2012) at this conference.

BBSO has installed NST, and first light with adaptive optics (July 2, 2010) has been attained in the Coude Lab. A first light image of granulation is shown in Figure 5E. The new telescope offers a significant improvement in ground-based high angular resolution capabilities, and will substantially enhance our efforts to understand our dynamic star. See publications from NST Observations: http://www.bbso.njit.edu/. At present NST is fully operational and it also acts as a pathfinder for future off-axis telescope projects to improve the maturity of evolving technologies (Figure 5). In particular the $1.7 \mathrm{~m}$ off-axis primary mirror (Figure $5 \mathrm{C}$ ) was polished by SOML and used as a prototype (proof-of-concept) for the $6 \times 8.4 \mathrm{~m}$ parabolic off-axis mirrors for the GMT (as discussed later). The NST M1 was figured to a final residual error of $16 \mathrm{~nm}$ RMS. 


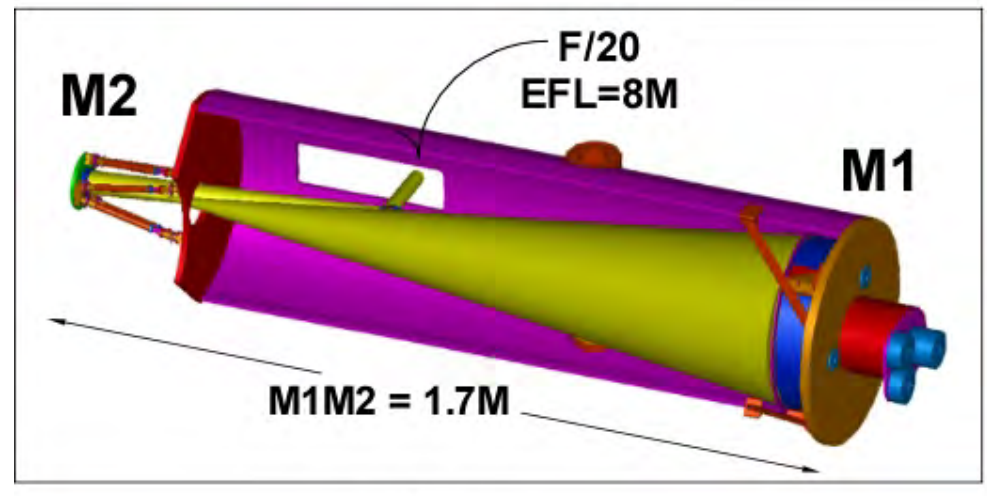

(A)

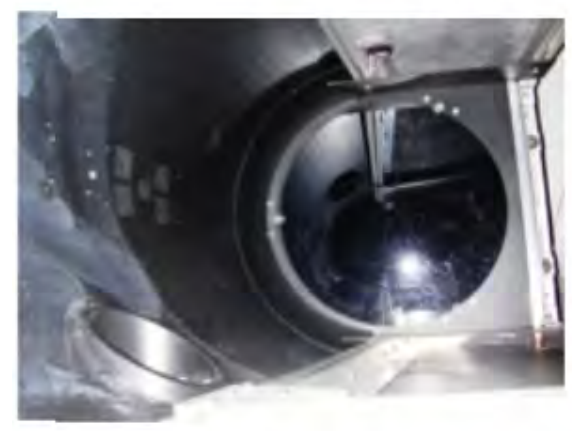

(C)

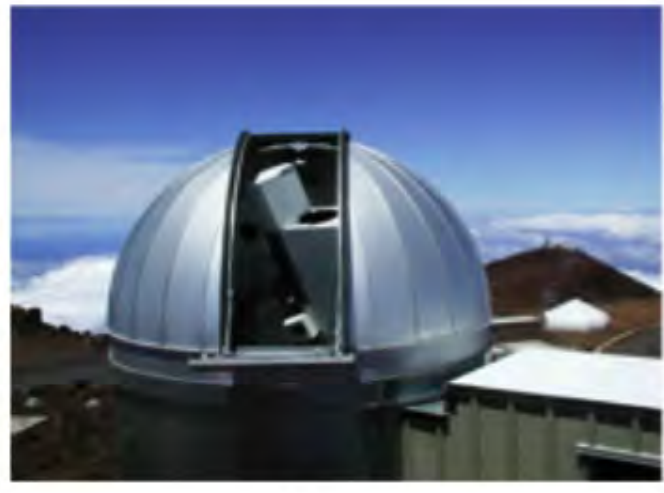

(B)

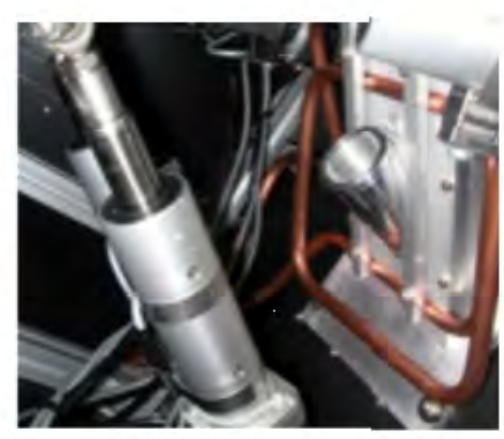

(D)

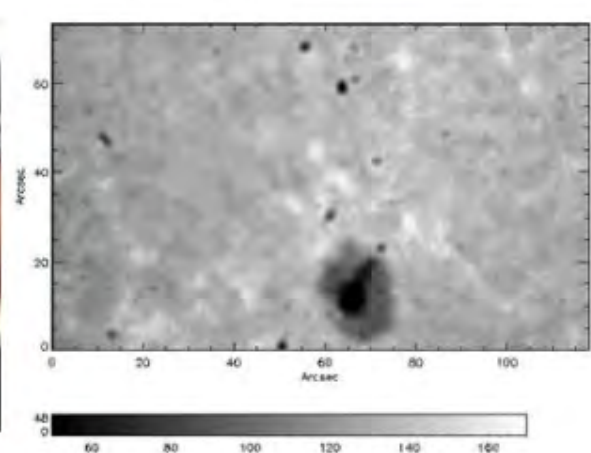

(E)

Figure 4 - SOLAR C - (A) The off-axis Gregorian system with a $0.45 \mathrm{~m}$ diameter primary mirror. The prime focus plate scale is 120 arc-sec/mm with a focal ratio of f/3.8. The system is a F/20 is diffraction-limited over an 18 arc-minute field at 1 micron. (B) SOLAR $\mathrm{C}$ Dome above looking west southwest. A look at the construction of SOLAR-C showing in $(\mathrm{C})$ the $0.5 \mathrm{M}$ off-axis primary mirror and in (D) the field stop cooling system. The first light was achieved in 2001, when the telescope captured the (E) sunspot imaging. (http://www.ifa.hawaii.edu/haleakalanew/solarc.shtml)

\section{OFF-AXIS TELESCOPES UNDERWAY}

Following the SOLAR-C and NST two projects has been proposed, funded and are underway: ATST and PLANETS.

ATST - The Advanced Technology Solar Telescope is a project of the National Solar Observatory operated by the Association of Universities for Research in Astronomy (AURA), under a cooperative agreement with the US National Science Foundation (NSF). ATST represents a collaboration of 22 institutions, reflecting a broad segment of the solar physics community. The construction phase of the project, to build the next generation ground-based solar telescope, is underway now. See McMullin et al (2012) at this conference. 


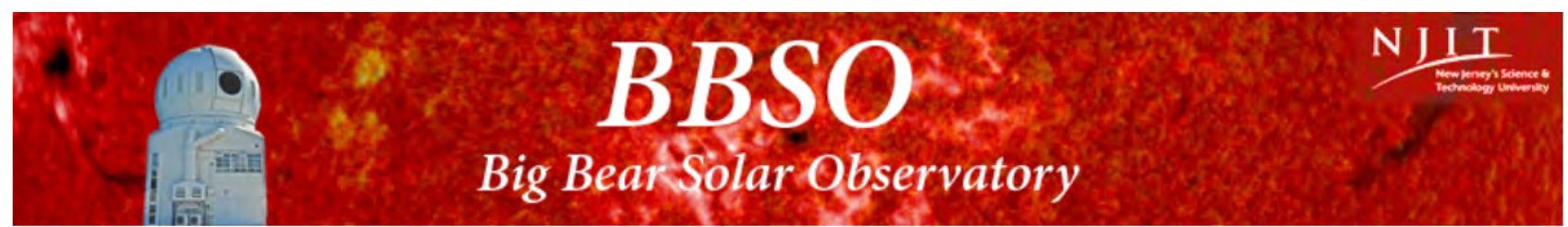

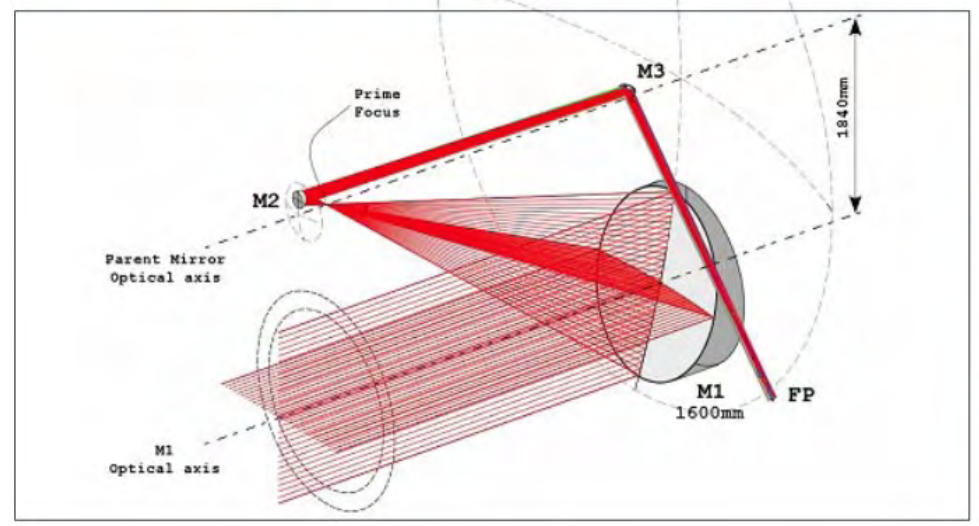

(A)

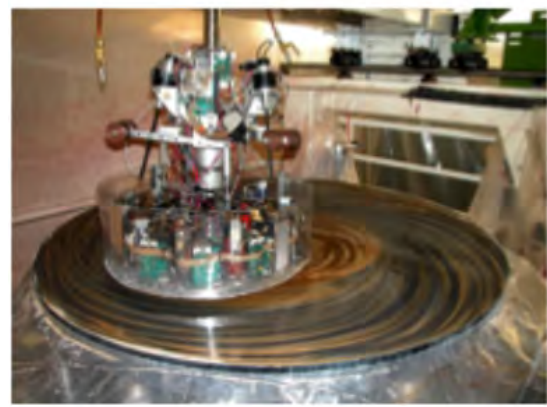

(C)

(D)

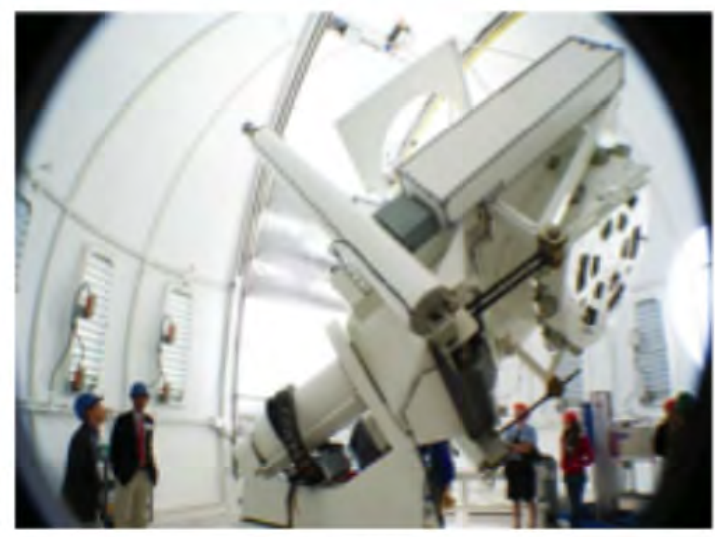

(B)

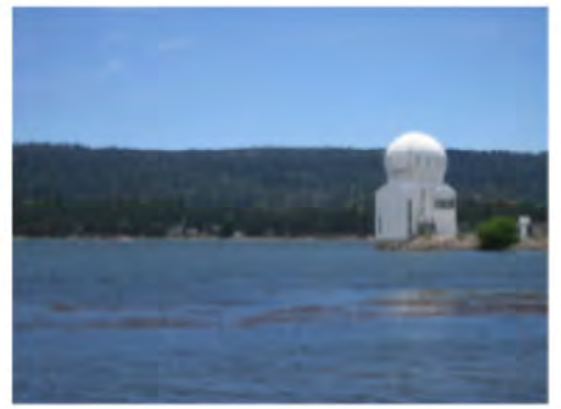

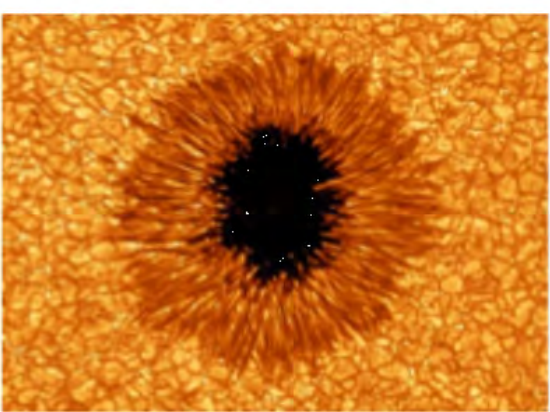

(E)

Figure 5 - NST - (A) The off-axis Gregorian system with a $1.7 \mathrm{~m}$ diameter F/2.4 primary mirror. The Gregorian focus is a F/52 $(\mathrm{EFL}=83.2 \mathrm{~m}$ ) with a plate scale $2.48 \mathrm{Arcsec} / \mathrm{mm}$. (B) The NST open telescope structure with an equatorial mount. The primary mirror is supported by 36 actuators that can blend out low-order aberrations, such as those due the gravity and/or thermal effects. M1 cell is clearly visible beneath the telescope structure. (C) The M1 being polished with $30 \mathrm{~cm}$ stressed lap at SOML. (D) The telescope is located in the lake which provides a natural inversion that minimizes the image blurring caused by atmospheric turbulence. (E) First Light with AO: Sunspot observed in TiO 706nm on July 2, 2010. A small sunspot (dark) surrounded by its penumbra (elongated fibrils). The penumbra is then surrounded by the Sun's unbiquitous granular field. The individual granules are the bright cells that can be as large as California. This picture was selected in 2010 as the most precise picture of the Sun (Ciel et Espace Magazine (France)). (http://www.bbso.njit.edu/)

The telescope is an off-axis Gregorian configuration based on an altitude-azimuth mount with independently-rotating Coudé platform. Configuration optimized across a 3 (minimum) to 5 (goal) Arcmin FOV producing image quality with seeing limited (no AO): under excellent seeing conditions, $<0.15$ arc-sec $@ \lambda=1600 \mathrm{~nm}$ and diffraction limited (with AO): under median seeing conditions, Strehl ratio $>0.3 @ \lambda=500 \mathrm{~nm}$. Scattered light performance goal is $<2.5 \times 10^{-6}, 1 / 10$ th solar radii from limb @ $\lambda=1000 \mathrm{~nm}$. Polarization accuracy $>10^{-4}$ of continuum intensity and sensitivity of $10^{-5}$ continuum intensity (limited by photon statistics). Coronagraphic mode optimized over near infrared through infrared. 


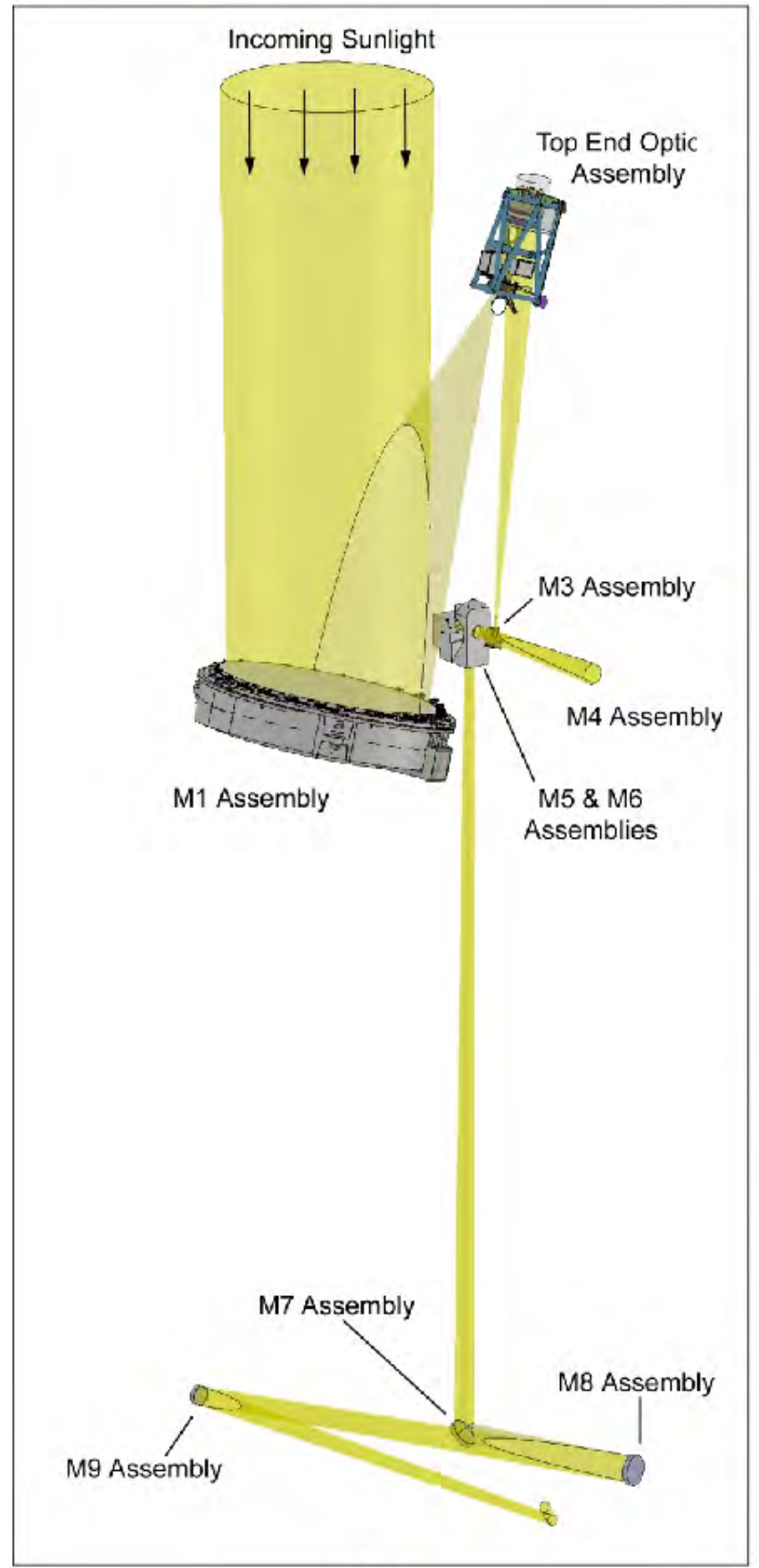

(A)

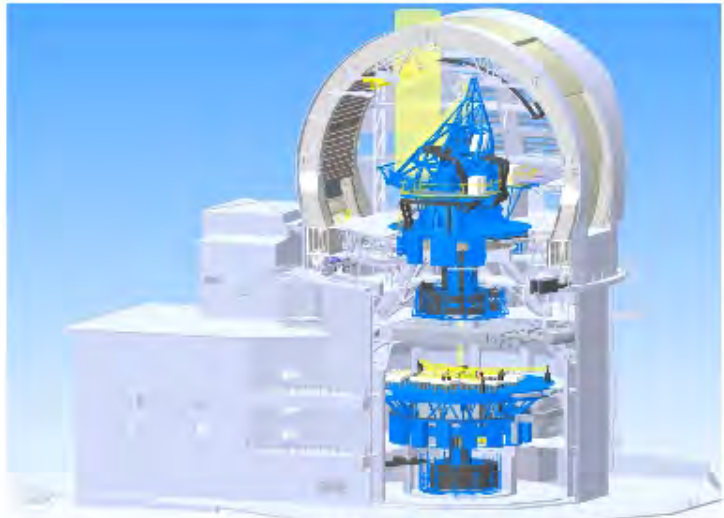

(B)

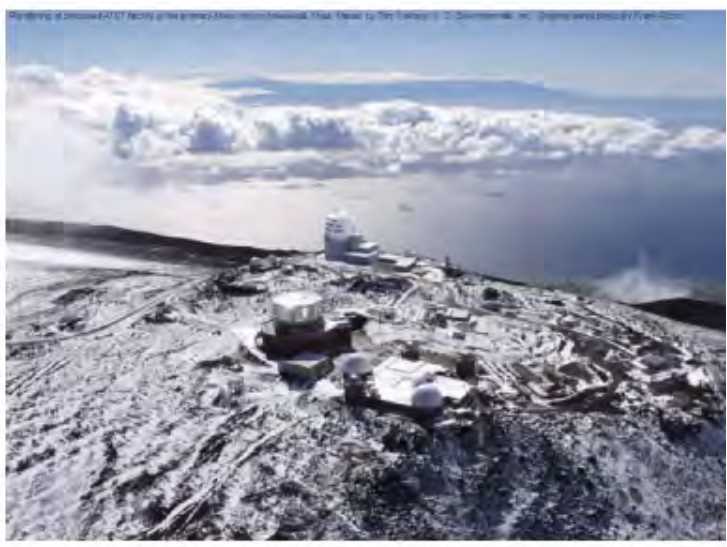

(C)

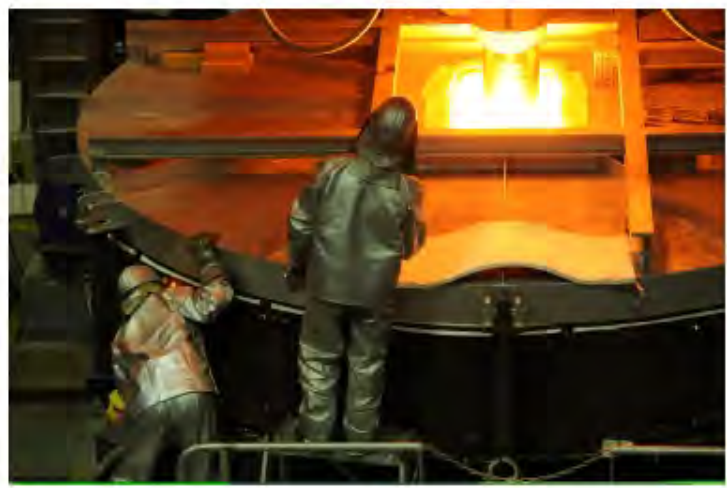

(D)

Figure 6 - The ATST project: (A) Schematic telescope mount assembly showing the independently-rotating two-story Coudé platform. (B) The optical core of off-axis Gregorian concept up to Coudé lab. (C) Cutaway facility view. (D) Casting of M1 blank begin in June of 2012 at SCHOTT AG for delivering in 2014, at which time SOML will begin polishing to the final specifications. (E) Render of proposed ATST facility on Haleakalã. More information: http://atst.nso.edu/.

The $4 \mathrm{~m}$ off-axis telescope ATST project is the latest in a sequence of mature smaller off-axis telescopes like SOLAR-C and NST. It will define a new state-of-the-art in terms of off-axis telescope and adaptive optics technologies in order to deliver unprecedented abilities to view details and to advance our understanding of the Sun. 
PLANETS - This off-axis telescope represents the interests of many institutions and research groups across the globe: Tokoku University (Japan); Institute for Astronomy, University of Hawaii (HI, USA); Kiepenheuer Institute for Solar Physics (Germany); The National University of Mexico (Mexico); Laboratory for Atmospheric and Space Physics, University of Colorado (CO, USA).
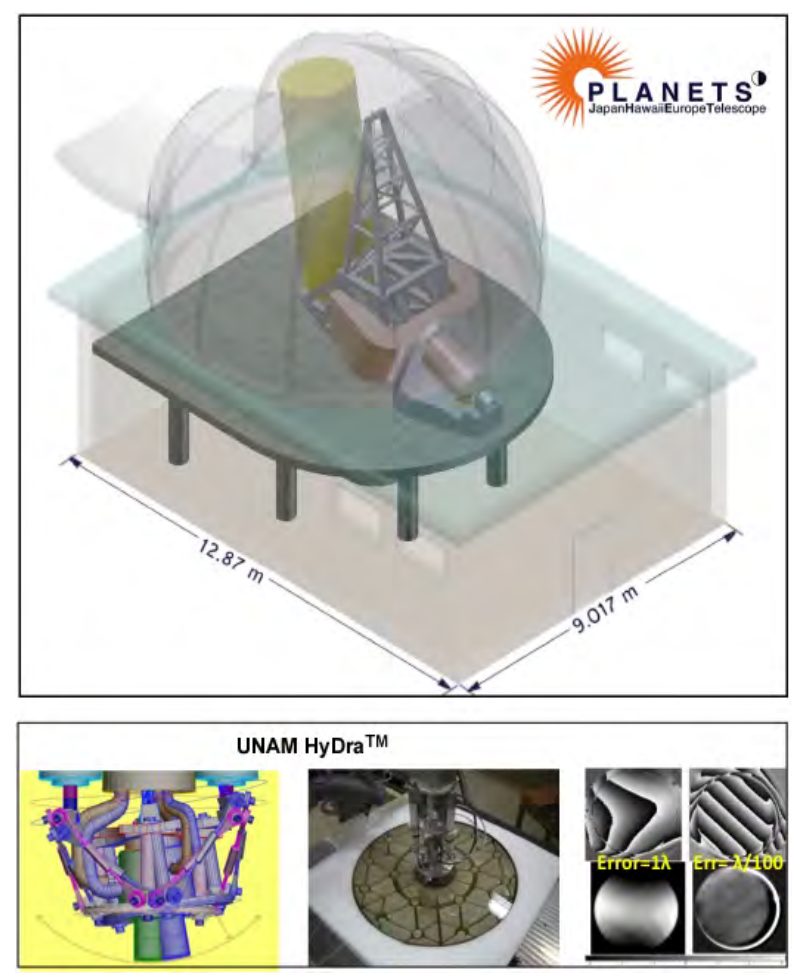

Figure 7 - The PLANETS project: A 1.9m off-axis telescope optimized to deliver high-dynamic range imaging, imaging polarimetry, aperture polarimetry, spectropolarimetry and coronagraphy assisted by adaptive optics. Telescope mirrors will be highly polished making use of UNAM HyDra ${ }^{\mathrm{TM}}$ (Hydrodynamic Radial Polishing Tool) technique. At the bottom-left corner the Hydra mounted on a hexapod with load cell zero-force operation. At bottom-middle Hydra polishing an $84 \mathrm{~cm}$ diameter mirror. It was demonstrated $\lambda / 100$ polishing on several optics.
The PLANETS $1.9 \mathrm{~m}$ off-axis telescope will be similar in design to the $1.6 \mathrm{~m} \mathrm{NST}$. This telescope will be ideal for coronagraphy and other techniques requiring stable optical path as it will be seeing limited with very low instrumental scattered light. By combining expertise from various fields - coronagraphy and high contrast imaging from solar physics, polishing, polarimetry and adaptive optics from astronomical communities and the experience of each institutional partner, this telescope will make significant advances in several fields. The telescope will be constructed on Haleakala, a $3000 \mathrm{~m}(10,000 \mathrm{ft})$ volcano on the island of Maui, HI with excellent weather and seeing.

The telescope will be highly polished to minimize diffuse scatter from mirror roughness - a major source of scattering at large angles. One of project partners UNAM has developed a new tool HyDra ${ }^{\text {TM }}$ (Hydrodynamic Radial Polishing Tool) to polish extremely smooth surfaces. The group has recently demonstrated 1/100th of a wavelength polish on several optics. Figure 7 at bottom shows an $84 \mathrm{~cm}$ diameter mirror been polished making use of $\mathrm{Hydra}^{\mathrm{TM}}$. This polish will further reduce the diffuse scattered light component from the telescope increasing the dynamic range of the telescope.

The PLANETS will make use of adaptive optics, which can be used to suppress atmospheric distortion and scattering. By combining adaptive optics with other techniques, greater contrast enhancement and dynamic range are possible. 


\section{OFF-AXIS TELESCOPE NEW CONCEPT}

Lessons from this review have shown that optical technology now enables the possibility of building a telescope that maximizes the point spread function (PSF) core energy while minimizing the scattered light flux in the wings of the PSF. These are the key requirements for achieving high angular and photometric dynamic range that had contributed heavily to the concept of the High Dynamic Range Telescope (HDRT). This new concept combines the tool of (1) an exquisite sensitivity for observing faint astronomical objects in the near environment of bright sources and (2) to provide wide-field observations.

The HDRT design study responds to the important question, "How should mirror segments in a large optical/infrared astronomical telescope be arranged to maximize the image clarity?" by allowing for the best possible adaptive optics system. A fundamental concern arises from the mirror segment edges. Mirror edges are difficult to polish accurately and the relative large-angle diffracted energy from a point source increases with the perimeter-to-area ratio of the pupil element. Straight-line edges in the pupil also tend to diffract light to larger angles than curved segments. It follows that we can minimize the effect of wavefront errors generated by mirror edges and large-angle diffraction by making the mirror segments as large as possible.

As described by Kuhn et al (2001), circular segments maximize this ratio and are the building blocks for the unobstructed telescope pupil design of the HDRT. This approach minimizes light scattering and the number of edge supports that are needed to actively control each mirror surface, and it will lead to images of unrivaled clarity. More specifically it was showed that a hexagonal pattern of circular mirrors with a spacing 4 percent larger than the diameter of each mirror nearly reproduces the resolution and performance of a single large mirror of that diameter. This "magic" ratio describes the placement of 8-meter mirrors in the HDRT pupil plane. Since its building blocks are now (2012) "conventional" 8-meter mirrors it is straightforward to design an adaptive optics system. This technology solves one of the leading problems facing large telescopes: how to make an AO system work on a large telescope.

Figure 8 shows HDRT concept layout as it was proposed (2000) as a replacement for the CanadaFrance-Hawaii Telescope (CFHT) on Mauna Kea. The HDRT concept achieves an effective light collecting area of an unobstructed $15.9 \mathrm{~m}$ diameter telescope by using six $6.5 \mathrm{~m}$ off-axis mirror segment. Despite the fact that it was showed that $8.0 \mathrm{~m}$ mirror segments are optimal, the choice of $6.5 \mathrm{~m}$ off-axis segment was determined (1999) by polishing cost concerns (like the dimensions of the fabricator optics tower), today we know that the limit is $8.4 \mathrm{~m}$ off-axis segments (SOML, 2011). This optical configuration allows three distinct optical operating modes from the same facility: (A) a full AO compensated F/15 coronagraphic telescope with a diffraction limited FOV at least 10 Arcsec and resolution of 12 milliarsec at $1000 \mathrm{~nm}$, (B) a moderate F/15 mode optimized across a $3 \times 3$ Arcmin FOV and (C) a wide-field F/1.3 mode optimized across a 1x1 degree FOV.

Another fundamental advantage of the HDRT is the versatility of its "open" design. Since its mirrors do not actually touch each other, it is possible to design an efficient mechanical system that both supports the mirrors and instruments, and allows for great flexibility in adding secondary optics. For example, the HDRT will be unique in its ability to operate in a wide-field mode (perhaps with a field of view as large as 3 degrees) while serving as a narrow-field imaging and coronagraphic telescope. 


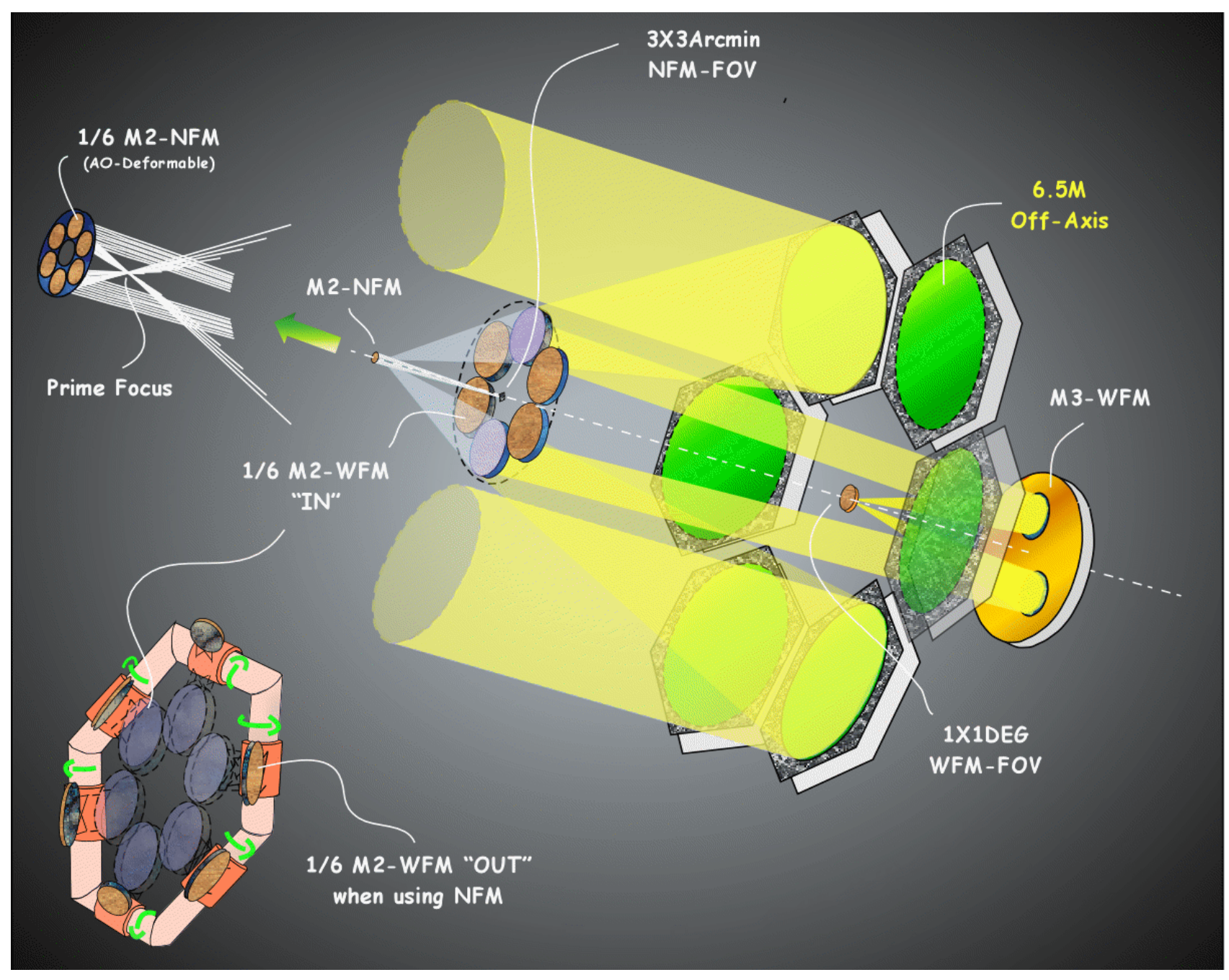

Figure 8 - The full HDRT optical layout is shown here. At the bottom-left corner of the figure, M2-WFM is composed of six subaperture mirrors and they can be separately folded up or down - like petals on a flower - out of the way of the NFM light path. At the top-left of the figure we see the six narrow-field subaperture mirrors that are $140 \mathrm{~mm}$ in diameter, and the unobstructed prime focus.

The HDRT concept was based on a new concept - the New Planetary Telescope (NPT) - of an offaxis design for a single $6.5 \mathrm{~m}$ telescope optimized for high angular resolution, high sensitivity in the optical and thermal infrared, and high photometric dynamic range. The concept with the same primary mirror allows several modes of observation: (A) a narrow-field f/15 mode optimized across a 2 X2arcmin square FOV, and (B) a wide-field f/3 mode, optimized across a 2 degree FOV. The NPT was devised as a replacement for the NASA Infrared Telescope Facility, (Moretto and Kuhn, 1999).

The 25m Giant Magellan Telescope (GMT) has partly adopted the HDRT concept design. The telescope's primary mirror will consist of seven $8.4 \mathrm{~m}$ segments, forming a single optical surface with a collecting area equivalent to a $22 \mathrm{~m}$ telescope and the resolving power of a $24.5 \mathrm{~m}$ aperture. Each segment is an $8.4 \mathrm{~m}$ diameter borosilicate honeycomb sandwich mirror similar to the $6.5 \mathrm{~m}$ and the $8.4 \mathrm{~m}$ mirrors in use at large telescopes in Arizona and Chile. They are the largest segments that can be made, guaranteeing a smooth wavefront over the $8.4 \mathrm{~m}$ subapertures. 

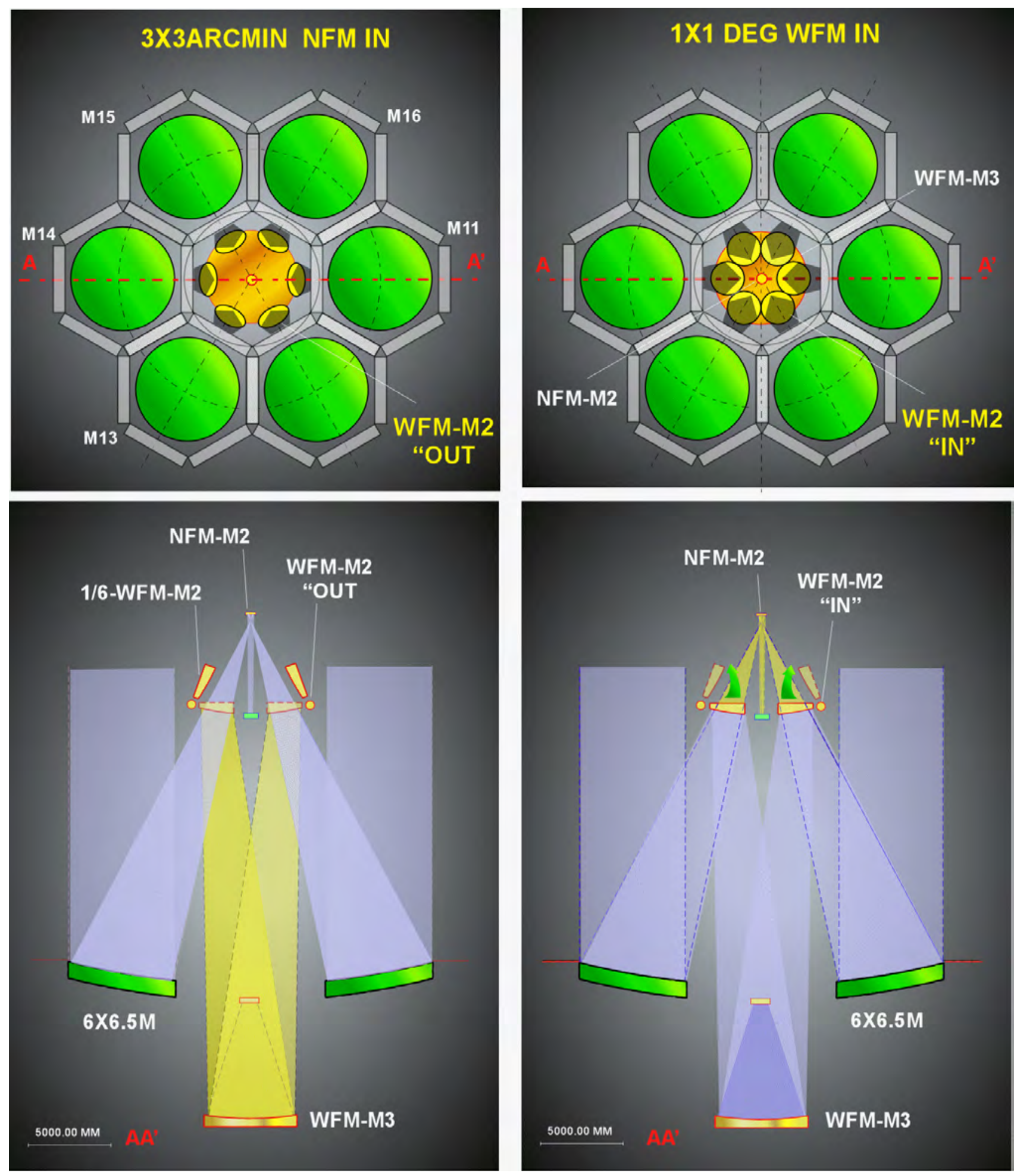

Figure 9 - The permanently mounted HDRT design combining WFM +NFM. The distance from the vertex of WFM-M3 to WFM-M2 is $24.5 \mathrm{~m}$ and the vertex distance from NFM-M2 to its FP is $6 \mathrm{~m}$. The distance from M1 vertex to WFM-M2 is $15.5 \mathrm{~m}$ and the M1 vertex to NFM-M2 is $22.4 \mathrm{~m}$. The NFM design produces a subaperture-M2 mirrors of $140 \mathrm{~mm}$ diameter. The diameter for the symmetrical WFM-M3 is $7.0 \mathrm{~m}$ and the subaperture mirrors are $2.34 \mathrm{~m}$. 
As the HDRT concept the GMT's secondary mirror is segmented to match the primary, with seven $1.1 \mathrm{~m}$ pieces. The small, agile secondary segments will perform the fine alignment for each $8.4 \mathrm{~m}$ mirror. They will be deformable and enable adaptive optics, phasing, and the coherent combination of the seven subapertures.
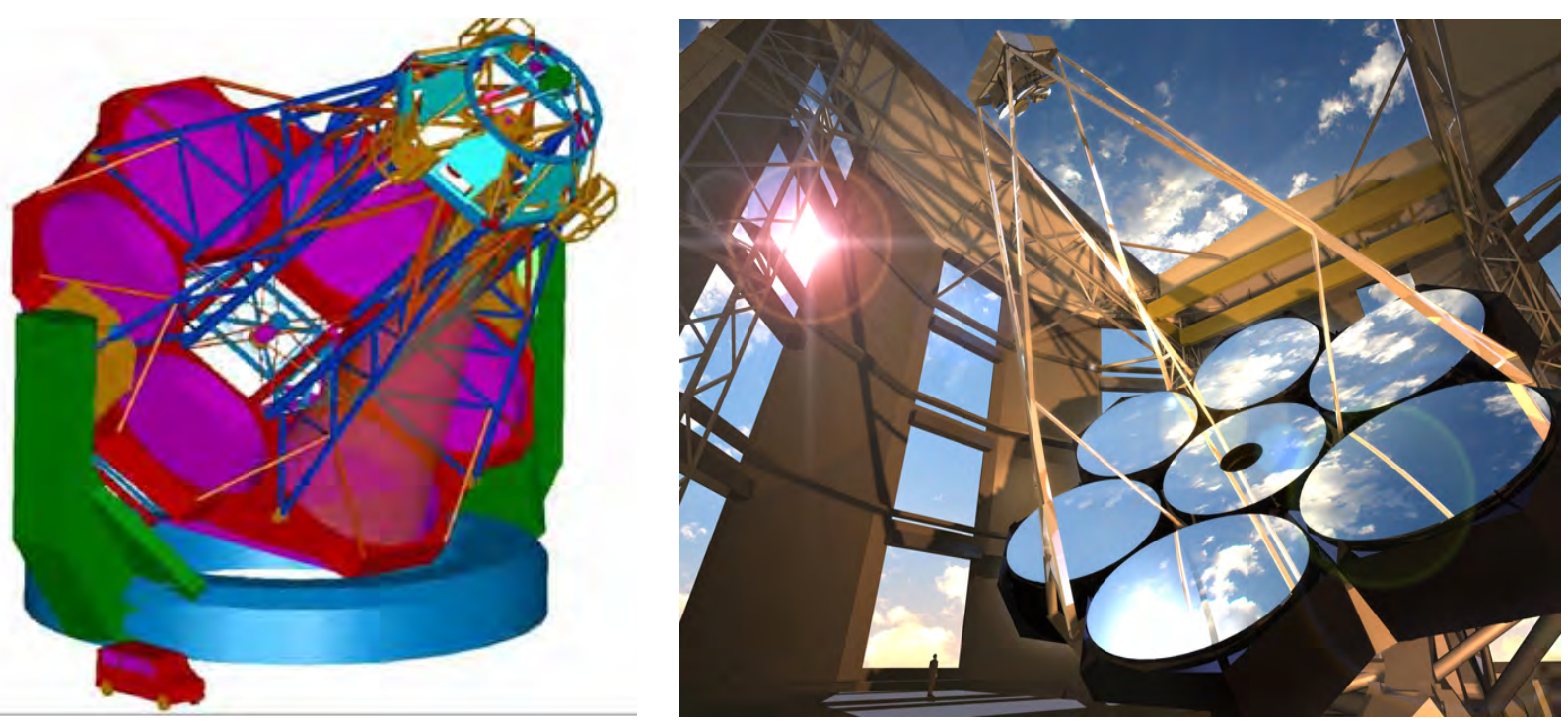

Figure 10 - At the left part of the figure the full HDRT and at the right side the GMT optical support systems for comparison. Note at the top-right corner of the HDRT figure is the M2-WFM support structure composed of six subaperture mirrors and they can be separately folded up or down out of the way of the NFM light path.

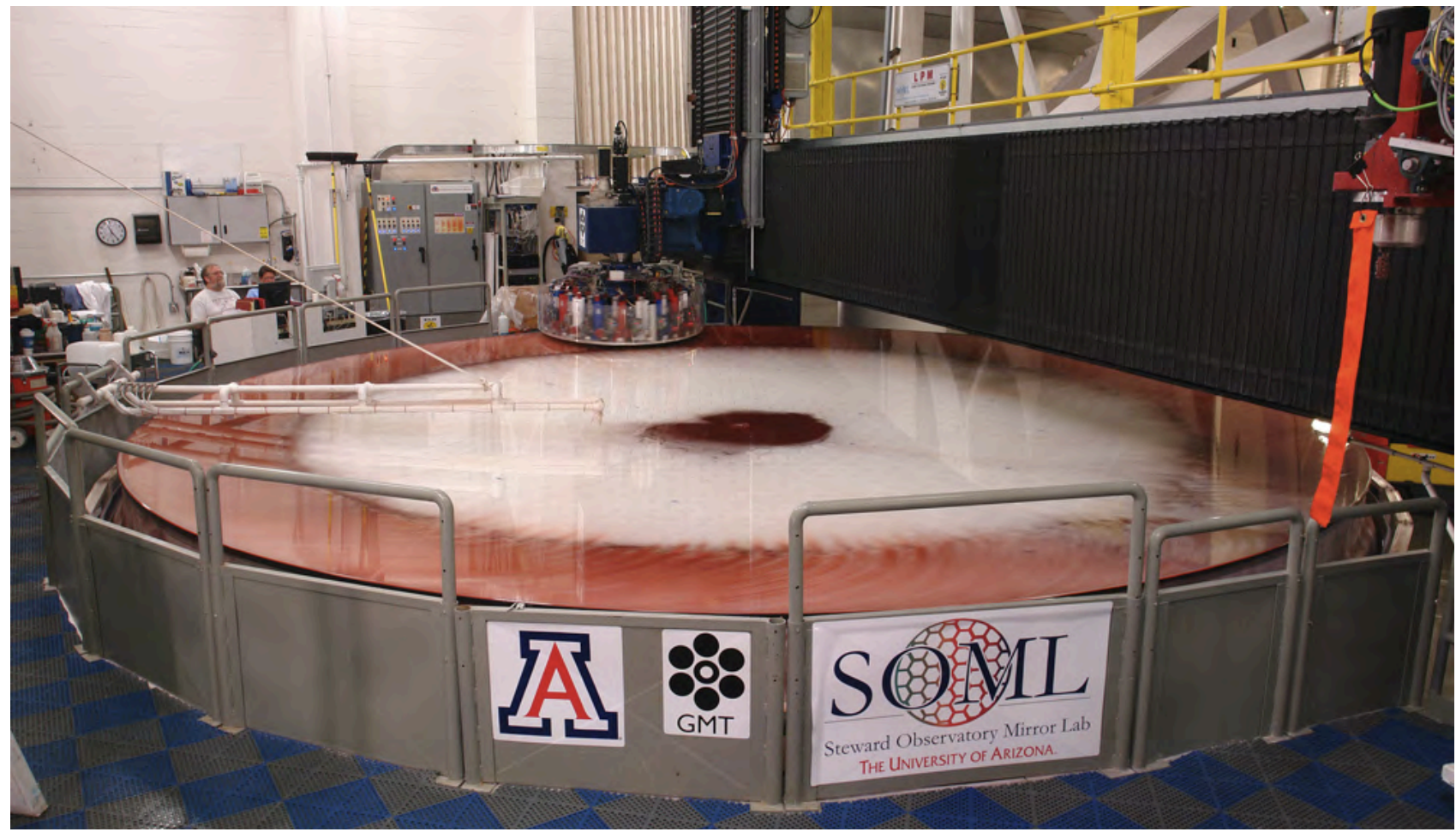

Figure 11 - The GMT's first 8.4m off-axis segment mirror polishing with stressed lap technique developed for high aspheric surfaces by SOML (2011). 
SOML has successfully finished the polishing of the first GMT's 8.4m off-axis segment. Figure 11 shows the polishing with stressed-lap technique, which was developed for the highly aspheric $8.4 \mathrm{~m}$ optical surface and make use of technology readiness gained from the $1.7 \mathrm{~m}$ off-axis prototype and primary mirror for the NST, which is a single PM, one-fifth scale version of the GMT. For the GMT, a new test tower and the principal optical test setup making use of advances in laser metrology and computer-generated holograms. (See Martin et al., 2012 at this conference).

Sagem (France), through its Reosc department has also started polishing and testing prototypes segments for the ELT-M1 (see Rodolfo et al., 2012, at this conference).

\section{OFF-AXIS TELESCOPE NEW CONCEPT FOR ANTARCTICA}

The Antarctic plateau offers exceptional atmospheric and environmental conditions for astronomical observations over a wide range of wavelengths and uniquely favorable to infrared astronomy. Exceptional low sky brightness throughout the near- and mid-infrared and a telescope facility complying with the highest possible dynamic range for photometry, angular resolution and the widefield leads to the possibility of a modest-sized $2 \mathrm{~m}$ off-axis telescope achieving comparable sensitivity to that of a larger ground-based 8-10m class telescope or a same sized space-based ones.

Moretto et al., 2012 (at this conference) have proposed a baseline design concept for Dome $\mathrm{C}$ which relies on a 2.5 meter unobstructed aperture telescope configuration which should produce a F/8 system optimized over an 1x1 Deg FOV as shown in Figure 12.
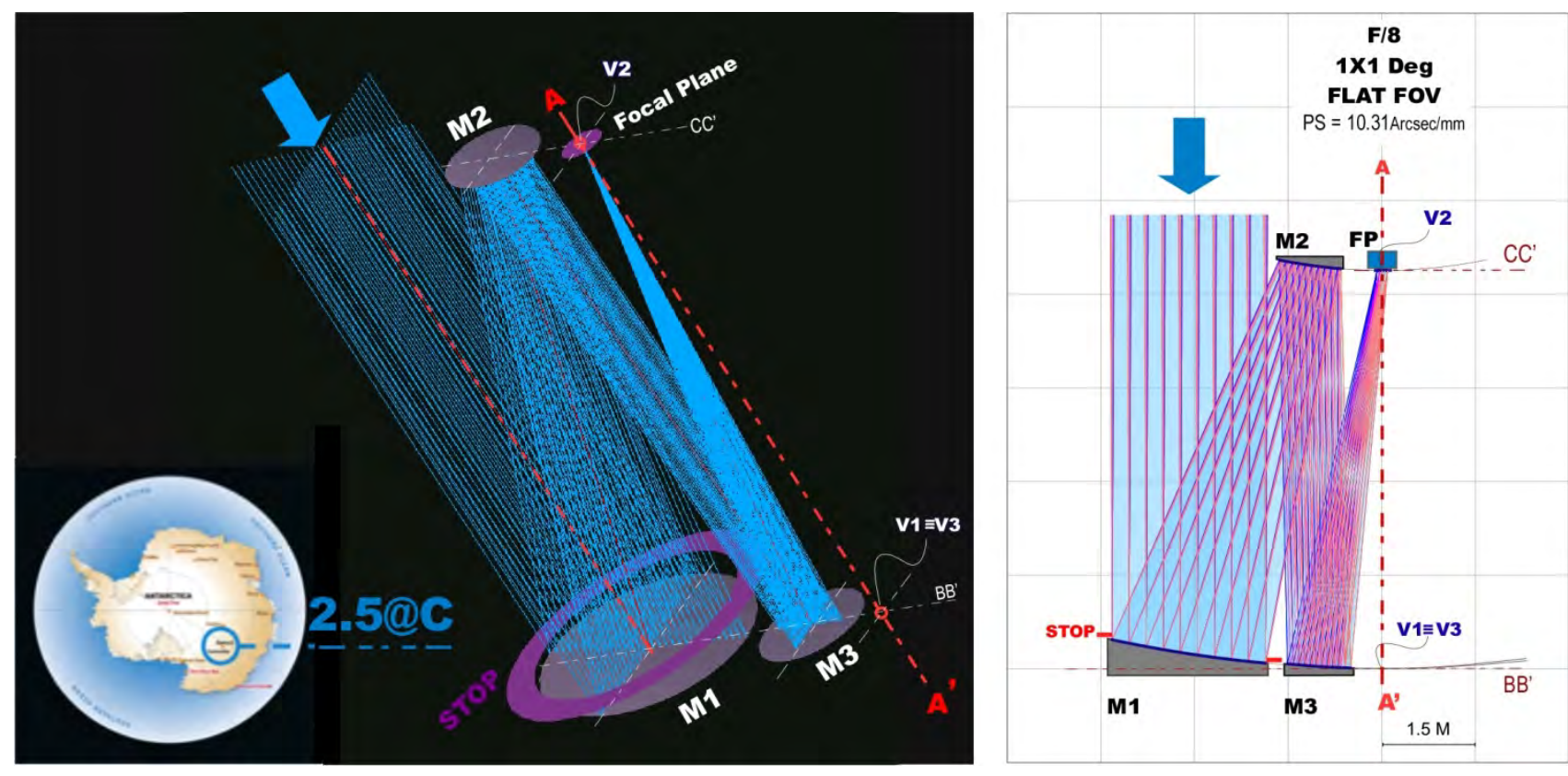

Figure 12 - The 2.5@C an off-axis telescope for Dome C. A 2.5 meter unobstructed aperture M1 yielding in a F/8 system optimized over a 1x1 Deg FOV. AA' is the parent mirrors M1, M2 and M3 optical axis. M1 and M3 vertexes V1 and V3 are coincident as well as for M2 (V2) and FP. Note that the primary mirror M1, the secondary M2 and the tertiary M3 are not tilted mirrors but are a decentered piece of concentric system, preserving its bi-lateral symmetry. 
The properties of the atmosphere above the Antarctic Plateau are known to be unique on the Earth's surface. Atmospheric turbulence is concentrated in a thin layer of a few tens of meters, the sky opacity, particularly in the infrared, is considerably reduced and the thermal infrared sky background radiation is lower by a factor of 10 to 20 in the $2-3 \mu \mathrm{m}$ windows. The combination of these advantages makes a 2 meter class telescope comparable to an 8 meter one in a conventional site in terms of sensitivity. The Antarctic Plateau is thus likely to become in the coming decades a new major platform for advanced astronomical observations. In the short/medium term, it is expected that synoptic infrared imaging and spectro-imaging surveys exploring the temporal dimension, from Antarctica could play an essential role in the context of the future large ground based and space projects such as E-ELT, JWST, LSST, EUCLID, GAIA.

A medium/large aperture telescope on the Antarctic Plateau have the potential to undertake tasks previously thought to be possible only in space, for example the imaging and crude spectroscopy of Earth-size extra-solar planets (Angel, Lawrence and Storey, 2004).

\section{CONCLUSIONS}

Many areas of modern astrophysics are not flux-limited but are rather dynamic-range limited. Simply collecting more photons will not solve the problem - for these topics, we don't need bigger telescopes, we need better telescopes set up at exceptional sites - we need off-axis telescopes! From this review it is evident that with the maturity of technology the feasibility of off-axis telescopes is a reality.

Many areas of modern astrophysics are flux-limited! Optical technology dictates that single mirrors cannot be made large enough for the next generation of telescopes! How should mirror segments in a large optical/infrared astronomical telescope be arranged to maximize dynamic range? We presented here the HDRT concept which addresses all of these questions to obtain a very efficient and versatile large or extremely large telescope. Despite the loss in circular symmetry, future telescopes may realize significant advantages in large-angle scattered light performance,

reduced telescope emissivity, better core PSF fidelity from AO systems, and improved out-of-field light rejection properties. 


\section{REFERENCES}

[1] Angel, R., Lawrence, J., Storey J., "Concept for a second Giant Magellan Telescope (GMT) in Antarctica," Proc. SPIE 5382, 76-84 (2004).

[2] Goode, P. R. and Cao, W., "The 1.6m new solar telescope (NST) in Big Bear," Proc. SPIE 8440, (2012).

[3] Kuhn, J.R., Howley, S.L., "Some Astronomical Performance Advantages of Off-Axis Telescopes," PASP, 111, (1999).

[4] Kuhn, J.R., Moretto, G., Racine F., Roddier F., Coulter, R., “A Large Aperture, High Dynamic Range Telescope Concept,’ PASP, 113 (2001).

[5] Martin, H. M., Allen, R. G., Burge, J.H., et al., "Production of 8.4m segments for the Giant Magellan Telescope" Proc. SPIE 8450, (2012).

[6] Moretto, G., Epchtein, N., Langlois, M., Vauglin, I., "2.5@C- An Off-Axis Telescope Concept for Antarctic Astronomy," Proc. SPIE 8444, (2012).

[7] Moretto, G. and Kuhn, J.R., "Off-Axis System for 4m Class Telescope, " Applied Optics, 37 No 16, (1998).

[8] Moretto, G. and Kuhn, J.R., "Optical Performance of the 6.5-m Off-Axis New Planetary Telescope," Applied Optics, 39 No. 16, (2000).

[9] Rodolfo, J. F. et al., "Prototypes segment polishing and testing for the ELT M1, " Proc. SPIE 8450, (2012). 